\title{
ON WEIGHTED HARDY INEQUALITY WITH TWO-DIMENSIONAL RECTANGULAR OPERATOR - EXTENSION OF THE E. SAWYER THEOREM
}

\author{
Vladimir D. Stepanov* And Elena P. Ushakova
}

Abstract. A characterization is obtained for those pairs of weights $v$ and $w$ on $\mathbb{R}_{+}^{2}$, for which the two-dimensional rectangular integration operator is bounded from a weighted Lebesgue space $L_{v}^{p}\left(\mathbb{R}_{+}^{2}\right)$ to $L_{w}^{q}\left(\mathbb{R}_{+}^{2}\right)$ for $1<p<q<\infty$, which is an essential complement to E. Sawyer's result [13] given for $1<p \leqslant q<\infty$. Besides, we demonstrate that the E. Sawyer theorem is actual if $p=q$ only, for $p<q$ the criterion is the finiteness of the Muckenhoupt-type constant. The case $q<p$ is also discussed. Mathematics subject classification (2020): 26D10, 47G10.

Keywords and phrases: Rectangular integration operator, Hardy inequality, weighted Lebesgue space.

\section{REFERENCES}

[1] S. BARZA, Weighted multidimensional integral inequalities and applications: Doctoral Thesis 1999:30, Luleå University of Technology, Department of Mathematics, Luleå , Sweden, 1999.

[2] A. Gogatishvili, A. Kufner, L.-E. Persson And A. Wedestig, An equivalence theorem for integral conditions related to Hardy's inequality, Real Anal. Exchange 29, 2 (2003/04), 867-880.

[3] V. KokilashVili, A. Meskhi AND L.-E. Persson, Weighted norm inequalities for integral transforms with product kernels, Nova Science Publishers, New-York, 2009.

[4] A. Kufner, L. Maligranda And L.-E. Persson, The Hardy inequality. About its history and some related results, Vydavatelský Servis, Plzeň, 2007.

[5] A. Kufner, L.-E. Perss SON AND N. S AmKo, Weighted inequalities of Hardy-type, World Scientific Publishing Co. Inc., New Jersey, 2017.

[6] V. G. MAZ'JA, Sobolev spaces, Springer Series in Soviet Mathematics, Springer-Verlag, Berlin, 1985.

[7] A. MesKHI, A note on two-weight inequalities for multiple Hardy-type operators, J. Funct. Spaces Appl. 3, 3 (2005), 223-237.

[8] B. Muckenhoupt, Hardy inequalities with weights, Studia Math. 44, 3 (1972), 31-38.

[9] L.-E. PERSSON AND V. D. STEPANOV, Weighted integral inequalities with the geometric mean operator, J. Inequal. Appl. 7 (2002), 727-746.

[10] L.-E. Perss ON, V. D. STEPANOV AND P. WALL, Some scales of equivalent weight characterizations of Hardy's inequality: the case $q<p$, Math. Inequal. Appl. 10, 2 (2007), 267-279.

[11] L.-E. Persson And E. P. Ushakova, Some multi-dimensional Hardy type integral inequalities, J. Math. Inequal. 1, 3 (2007), 301-319.

[12] D. V. Prokhorov, V. D. Stepanov and E. P. Ushakova, Hardy-Steklov Integral Operators: Part I, Proc. Steklov Inst. Math. 300, suppl. 2 (2018), 1-112.

[13] E. SAWYER, Weighted inequalities for two-dimensional Hardy operator, Studia Math. 82, 1 (1985), $1-16$.

[14] G. TomaselLI, A class of inequalities, Boll. Unione Mat. Ital. 2 (1969), 622-631.

[15] A. Wedestig, Weighted inequalities of Hardy-type and their limiting inequalities: Doctoral Thesis 2003:17, Luleå University of Technology, Department of Mathematics, Luleå , Sweden, 2003.

[16] A. Wedestig, Weighted inequalities for the Sawyer two-dimensional Hardy operator and its limiting geometric mean operator, J. Inequal. Appl. 4 (2005), 387-394. 